4. I.S. McLean, Astron. Astrophys. 134, 333, 1984.

5. J.O. Stenflo, D. Twerenbold, J.W. Harvey and J.W. Brault, Astron. Astrophys. Suppl. 54, 505, 1983.

6. J.0. Stenflo, J.W. Harvey, J.W. Brault and S. Solanski, Astron. Astrophys. $131,333,1984$.

7. LEST Foundation Annual Report 1983, p. 16.

8. J.0. Stenflo, Applied Optics 23, 1267, 1984.

9. F. Vakili, Proc. S.P.I.E. 445 (Instrumentation in Astronomy V), $484,1984$.

10. J.H. Apfel, Applied Optics $23,1178,1984$.

11. A.M. Title and W.J. Rosenberg, Proc. S.P.I.E. 307 (Polarizer Applications), $120,1981$.

12. Bull. Amer. Astron. Soc. 16, 1984.

13. J.A. Tyson and R.W. Lee, Proc. S.P.I.E. 290 (Solid State Imagers in Astronomy), $144,1981$.

14. R.G. Allen, J.R.P. Angel, Proc. S.P.I.E. 331 (Instrumentation in Astronomy IV), p. 259,1982 .

15. T. Korhonen, V. Piirola and A. Reiz, ESO Messenger no. 38, December 1984 , p. 20.

\title{
IV. Detector developments
}

There is little doubt that array detectors of some kind will eventually take over from the single-pixel detectors now used for precise work. The takeover, however, is a gradual process; more and more problems are encountered as the required precision is raised. Very broadly speaking, the best array detector practice has now reached $0.01 \mathrm{mag}$ in photometry and $0.1 \%$ in polarimetry $(0.001$ and $0.005 \%$ respectively for single detectors). Whenever this precision of the array detectors is sufficient and the multiplex advantage vital to the programme comtemplated, the array detectors are the indicated path to progress (see, for instance, Ref. 1).

Imaging a field rather than a single point with photometric precision introduces extra problems such as ghosts (Ref. 2); recording such images is further complicated by pixel-to-pixel variation of both gain factors and offsets. On the other hand, the availability of many parallel detectors offers the possibility of intercomparing the registrations of the same source by different element detectors and thus constructing an internally consistent calibrated image; progress in this field is considerable, but does not seem to have approached any fundamental limits yet and often suffers from ad hoc simplifications.

The problem, of course, is that in general both the zero offset and gain of elemental detectors are not identical and are functions of position of the element, wavelength, temperature, polarization, etc., and that some of these functional dependences are coupled. Decoupling and calibrating them in a relative sense are the keys to succesful photometry. The most "decoupled" situations would seem to be sky images in narrow bands excluding night-sky lines and spectrophotometry of single point objects, while "objective prism" images constitute the most complex situation and are least likely to yield reliable precision results (both these and broadband direct images are of course of great interest for their efficient acquisition).

Calibration sources are sometimes artificial, at other times the sky itself; in both cases it needs to be demonstrated that the spectral content and evenness of illumination are appropriate to the problem. From the point of view of our Commission, reports of array detector work should be read with the above considerations in mind.

To get an impression of the problems involved and the complexity of the solutions, good "student texts" are 34.002.043, Ref. 7 and Ref. 8 (the advanced student may proceed to Ref. 15, p. 2, Refs. 4, 5 and 6, probably to his complete bewilderment). The fundamental solution to our problems will be homogeneous array detectors, but for the moment this is wishful thinking at almost all levels of precision. 
The largest collection of papers on electronic area detectors is to be found in Refs. 4, 5, 6 and 15; modern photographic measuring machine practice is also very relevant (e.g. 33.012.059). The CCD for the Space Telescope's Wide Field \& Planetary Camera is one of the most fully documented devices (Ref. 3, 33.035.098 or 33.035.122, 34.157. 148 and lately Ref. 10, p. 477, where detection of 26th magnitude on the Hale 5-metre telescope is reported). For photometry, one of the promising ways of using a CCD is the drift-scan method (Ref. 7, and Ref. 9 or Ref. 5, p. 137). In this technique, pixel inhomogeneities are averaged along a column and the sky itself is used for gain calibration; the limits to the technique and its inherent assumptions need to be systematically investigated for photometric use (as opposed to mere detection) and the merits compared to those of, for instance, a series of strongly overlapping discrete exposures.

Much work remains to be done before stellar photometry with array detectors becomes an everyday reality at the precision we are used to from single-detector photometry. However, the potential gains for statistical investigations and for spectro-photometric systems tailored to the application are enormous, so that the effort involved in raising the precision is fully justified. For the present, the focus of attention is on CCDs, because they are superior in faint object detection. For more precise work on brighter objects, they may not be the ideal type of array detector.

The following developments, though not yet "photometric", are of interest for their future promise:

-- A new photon-counting detector called Mepsicron is reported in Ref. 11. It has high uniformity of sensitivity and a large number of picture elements, coupled with adequate quantum efficiency and maximum count rate. It, and similar devices, may become an attractive alternative to the ubiquitous CCD.

-- Infrared array detector developments are reported in $34.034 .006,34.034 .061$, Ref's. 13, 14, a number of papers in Ref. 6 and in Ref. 10, p. 36, 232 and 238. The largest size reported so far is $64 \times 64$ elements.

- Ref. 10, p. 304 reports encouraging developments of an X-ray CCD, with spectral as well as spatial resolution and high quantum efficiency.

-- At Kitt Peak a comprehensive, transportable, array detector software system called IRAF is being developed, which includes photometric programmes (Ref. 10, p. 497). We shall all experience this system frequently in the future.

The problems of polarimetry with array detectors are different from those of photometry. Photometric precision is not of paramount importance in most applications; what is important is the ability to operate with polarization modulation at a few $\mathrm{Hz}$ or faster. Array polarimeters in the literature are mentioned in sec. III of this report; Ref. 3 of that section describes experience with the on-chip storage of the 2 charge images corresponding to the 2 polarised optical images. This promising method (Ref. $5, \mathrm{p} .76$ ) has not yet been brought to full operational use and may in fact be limited in scope (about $0.1 \%$ precision). An alternative possibility would seem to be a modified drift-scan camera; no developments in this direction have been reported.

The Mepsicron (Ref. 11) and similar high-readout-rate photon-counting detectors are attractive for polarimetry, although even 100 counts/sec from a pixel barely allows $0.1 \%$ polarimetric precision without further smoothing. A developmental detector Rubicon, based on the Digicon principle, may become important for spectropolarimetry (Ref. 12).

\section{REFERENCES}

1. A.J. Penny, Gemini no. 7, p. 5, 1983 and no. 12, p. 1, 1984.

2. C.G. Wynne, S.P. Worswick, C.M. Iowne and P.R. Jorden, Observatory 104, 23, 1984 .

3. M.M. Blouke, J.R. Janesick, J.E. Hall, M.W. Cowens, P.J. May, Opt. Eng. 22, $607,1983$.

4. Solid State Imagers for Astronomy, Proc. S.P.I.E. 290, 1981. 
5. Instrumentation in Astronomy IV, Proc. S.P.I.E. 331, 1982.

6. Instrumentation in Astronomy V, Proc. S.P.I.E. $4 \frac{335}{145}, 1984$.

7. P. Hall and C.D. MacKay, M.N.R.A.S. 210 , 979, 1984.

8. K.U. Ratnatunga and E.B. Newell, Astron. J. 89, 176, 1984.

9. J.T. McGraw, H.S. Stockman, J.R.P. Angel, H. Epps, T. Williams, Opt. Eng. 23, $210,1984$.

10. Bull. Amer. Astron. Soc. 16, 1984.

11. C. Firmani, L. Gutierrez, E. Ruiz, G.F. Bisiacchi, L. Salas, F. Paresce, C.W. Carlson, M. Lampton, Astron. Astrophys. 134, 251, 1984.

12. Th. Schmidt-Kaler, R. Rudolph, H. Tüg, I.A.U. Coll. 79 (Very Large Telescopes, their Instrumentation and Programs), 659, 1984.

13. J.F. Arens, G.M. Lamb, M.C. Peck, H. Moseley, W.F. Hoffmann, R. TreschFienberg, G.G. Fazio, Astrophys. J. 279, 685, 1984.

14. E.L. Dereniak, J.P. Britt, A.M. Fowler, R.R. Joyce, G. Boreman, W.S. Ewing, Applied Optics 23, 889, 1984.

15. State-of-the-Art Imaging Arrays and Their Applications, Proc. S.P.I.E. 501, 1984. 